\title{
Perinatal Survival of a Fetus with Intestinal Volvulus and Intussusception: A Case Report and Review of the Literature
}

\author{
Esohe Ohuoba, MD, $\mathrm{MPH}^{1, *}$ \\ Nikolaos Zacharias, $\mathrm{MD}^{1}$ \\ ${ }^{1}$ Department of Obstetrics and Gynecology, Baylor College of \\ Medicine, Houston, Texas \\ 2 Department of Molecular and Human Genetics, Baylor College of \\ Medicine, Houston, Texas \\ ${ }^{3}$ Department of Surgery, Baylor College of Medicine and Texas \\ Children's Hospital, Houston, Texas \\ "Esohe Ohuoba and Gary Fruhman contributed equally to this \\ manuscript.
}

Am J Perinatol Rep 2013;3:107-112.

\begin{abstract}
Address for correspondence Nikolaos Zacharias, MD, FACOG, Division of Maternal-Fetal Medicine, Department of Obstetrics and Gynecology, Baylor College of Medicine, One Baylor Plaza, Houston, TX 77030 (e-mail: nmzach@yahoo.com).
\end{abstract}

\begin{abstract}
Keywords

- fetal intestinal volvulus

- perinatal mortality

- intussusception

- intrauterine volvulus

Fetal intestinal volvulus is a rare life-threatening condition. Late diagnosis of volvulus contributes to high rate of morbidity and mortality. It has variable degrees of presentation and survival. Intrauterine volvulus may be complicated by intestinal atresia due to ischemic necrosis. To our knowledge, there are three reported cases of term fetal demise. We report a case of fetal intestinal volvulus with perinatal survival of the largest term infant described with this complication to date. The volvulus was associated with type $3 \mathrm{~A}$ jejunal atresia and intestinal pathology was noted on prenatal ultrasound. The infant was born via urgent cesarean delivery at $37^{6 / 7}$ weeks of gestation and underwent emergent exploratory laparotomy with resection of small bowel and primary end-to-end anastomosis. Intrauterine intestinal volvulus may be suspected on prenatal ultrasound but only definitively diagnosed postnatally. Signs of fetal distress and volvulus are rarely associated with reports of survival in the term fetus. We review reported cases of prenatally suspected volvulus in infants documented to survive past the neonatal period. As fetal volvulus and most intestinal atresias/stenoses manifest during the third trimester, we recommend that the limited fetal anatomical survey during growth ultrasounds at 32 to 36 weeks routinely include an assessment of the fetal bowel.
\end{abstract}

Fetal intestinal volvulus is a rare life-threatening condition. Late diagnosis of volvulus contributes to high rate of morbidity and mortality. It has variable degrees of presentation and survival. Intrauterine volvulus may be complicated by intestinal atresia due to ischemic necrosis.

\section{Case Report}

A 26-year-old $\mathrm{G}_{4} \mathrm{P}_{2103}$ woman presented to us for prenatal care in the mid-second trimester; her obstetrical history was significant for preterm birth in her first pregnancy (receiving weekly intramuscular injections of 17-hydroxyprogesterone caproate until 37 weeks to prevent recurrent preterm birth) and a cesarean delivery for lower genital herpetic lesions in her third pregnancy. The index pregnancy was complicated by maternal obesity (booking body mass index, 43), likely pregestational diabetes mellitus requiring insulin therapy, and latent herpes simplex virus infection (acyclovir suppression initiated at 36 weeks). Routine prenatal ultrasound was performed at $24^{2 / 7}$ weeks and was unremarkable (-Fig. 1). received

March 20, 2013

accepted after revision

June 5, 2013

published online

July 11, 2013
DOI http://dx.doi.org/

10.1055/s-0033-1349367. ISSN 2157-6998.
Copyright $\odot 2013$ by Thieme Medical Publishers, Inc., 333 Seventh Avenue, New York, NY 10001, USA. Tel: +1(212) 584-4662.
License terms

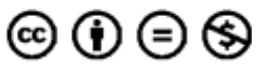




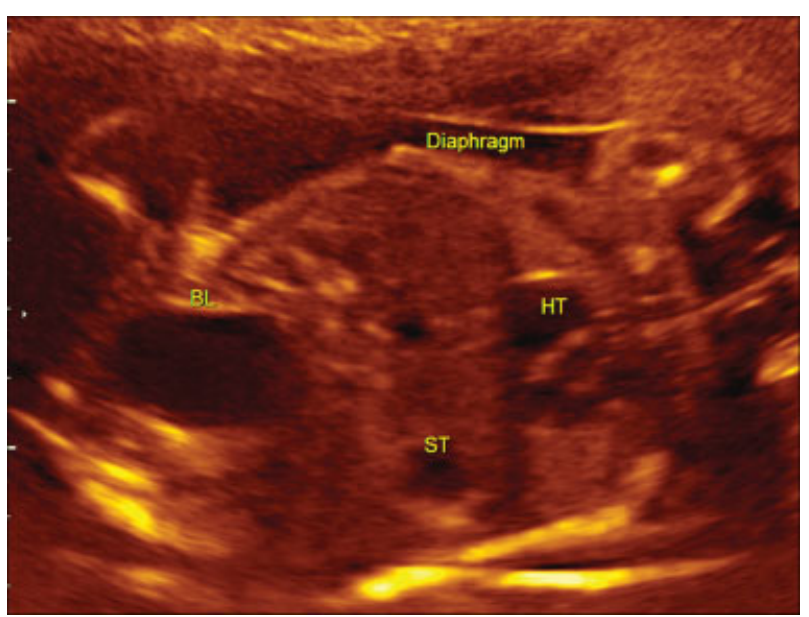

Fig. 1 Normal bowel at 24 weeks of gestation sonogram.

Follow-up ultrasound to assess interval growth at $36^{2 / 7}$ weeks revealed fetal mega-cisterna magna at 14 to $16 \mathrm{~mm}$ and striking tubular dilatation of the lower digestive tract concerning for complete intestinal obstruction. At that time, the differential diagnosis entertained the following pathological entities: jejunal atresia, ileal atresia, colonic atresia, meconium ileus, intestinal volvulus, imperforate anus, Hirschsprung disease (aganglionic megacolon), and meconium peritonitis. Umbilical artery velocimetry was normal, and neither Doppler interrogation of the middle cerebral artery nor brainsparing reflex activation find any evidence of fetal anemia (peak systolic velocity, 0.97 multiples of the median). Appropriate interval fetal growth was noted (60th percentile to 51st percentile between the two scans); the placenta and uterus were unremarkable and there was normal amniotic fluid volume (amniotic fluid index $=20.5 \mathrm{~cm}$ ). Interestingly, the standard abdominal circumference views were normal (-Fig. 2) and the intestinal dilatation was noted by the examining sonographer on a more caudal plane. Prolonged external fetal monitoring and testing on that day was reas-

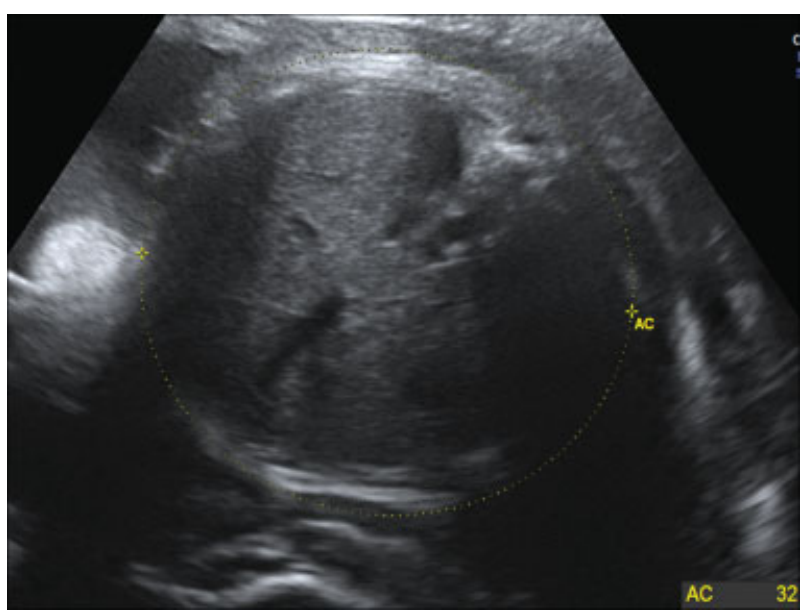

Fig. 2 Normal abdominal circumference at 36 weeks of gestation sonogram. suring (biophysical profile was 10/10) and cautious outpatient management was continued with twice weekly fetal surveillance until planned prelabor repeat cesarean delivery by $39^{0 / 7}$ weeks. Prenatal neonatology and pediatric surgical consultations were initiated.

The gravida presented to the obstetrical triage at $37^{6 / 7}$ weeks with decreased fetal movement for the past 2 days. Cardiotocography revealed minimal short-term variability and repetitive late decelerations that failed to respond to intrauterine resuscitation. A male infant of 3,353 $\mathrm{g}$ was born via urgent cesarean delivery due to non-reassuring fetal heart tones. At delivery, there was meconium-stained amniotic fluid and a single nuchal cord that was reduced without difficulty. Apgar scores were 1,5, and 7 at 1,5, and 10 minutes, respectively, and abdominal distention was immediately noted on neonatal examination.

The infant was found to have increasing abdominal tenderness and distension in the ensuing hours. An orogastric tube was placed and yielded dark and then bloody secretions. Initially, the neonate had spontaneous breathing but subsequently developed periods of apnea and desaturations requiring intubation. Umbilical venous blood showed $\mathrm{pH}$ of $7.22, \mathrm{PCO}_{2}$ of $44, \mathrm{Po}_{2}$ of 49 , bicarbonate of 17.2 , and base deficit of 9 (umbilical artery specimen clotted) and initial glucose was $<20 \mathrm{mg} / \mathrm{dL}$. Initial complete blood count revealed anemia with hemoglobin of $10.8 \mathrm{~g} / \mathrm{dL}$, platelet clumping with normal count $(183,000 / \mu \mathrm{L})$, and leukocytosis at 25,600 WBC/ $\mu \mathrm{L}$ predominantly lymphocytic (54\%). Because of pediatric concern for hypoxic ischemic encephalopathy (HIE), the infant was placed on a head-cooling protocol with induced hypothermia for brain protection.

Upon transfer to a quaternary neonatal intensive care unit for further specialized management, the neonate was found not meeting the HIE criteria and was gradually rewarmed in anticipation of exploratory laparotomy. Chest X-ray revealed ascites, KUB (kidney, ureter, and bladder X-ray) revealed small stomach bubble with no gas in bowel, and bedside abdominal ultrasound revealed multiple distended loops of small bowel filled with fluid. An urgent upper gastrointestinal series revealed the duodenojejunal junction to the right of the midline consistent with malrotation without radiographic evidence of obstruction. Emergent laparotomy was performed for suspicion of intestinal malrotation on the first day of life. Surgical exploration revealed markedly distended ischemic jejunum with about a 240-degree volvulus adjacent to an area of a "type $3 \mathrm{~A}$ " intestinal atresia (complete separation of the two blind bowel ends by a mesenteric defect). On the distal end of the atresia, there was a $57-\mathrm{cm}$ segment of small bowel-to-small bowel intussusception. Approximately $50 \mathrm{~cm}$ of bowel distal from the intussusception to the ileocecal valve was found to be patent. The entire colon appeared normal. The proximal bowel was distended and there was $17 \mathrm{~cm}$ of healthy proximal bowel from the orthotopic ligament of Treitz down to the area of bowel ischemia. Surgical resection of the small bowel volvulus and intussusception, with primary end-to-end small bowel anastomosis, were performed and the infant recovered well and was discharged from the hospital on 29 days of life. Pathological 
examination confirmed that the diffuse ischemic necrosis was consistent with volvulus, small bowel intussusception, fibrous adhesions, and small bowel atresia. No major complications occurred and at the 4-month postoperative followup, the infant was found to be doing well with appropriate interval growth. Two years later, the child continues to thrive. To our knowledge, no other case with prenatally suspected and postnatally confirmed intestinal volvulus, delivery at term, and prolonged follow-up with good outcome has been reported previously in the literature.

\section{Discussion}

Intestinal volvulus is a rare neonatal surgical emergency, and delays in diagnosis and timely intervention can contribute to high morbidity and mortality rates. Prenatal diagnosis was only suspected in 11 cases before $2001 .^{1}$ Between the 8 th and 10 th weeks of gestation, malrotation of the fetal gut occurs, when the elongating intestine returns to the abdominal cavity. $^{2}$ Between the 6 th and 10th weeks of fetal development, the midgut loop rotates 90 degrees counterclockwise around the axis of the superior mesenteric artery (SMA). This brings the duodenojejunal loop to the right and the ileocolic loop to the left side. During the 10 th to 11 th weeks, the intestines return to the fetal abdominal cavity. Both the proximal and distal loops undergo a total of 270 degrees of rotation. The duodenojejunal loop ends up posterior to the SMA and the ileocolic loop anterior to the SMA. The duodenojejunal junction lies in the left upper quadrant of the abdomen and the ileocecal junction in the right lower quadrant. $^{3}$

Volvulus with malrotation may result in variable degrees of ischemic necrosis. ${ }^{4}$ The bowel loops twist around the fetal mesenteric artery or its branches resulting in intestinal obstruction and vascular compromise, which can cause necrotic bowel perforation or even hemorrhagic ascites, anemia, and fetal demise. ${ }^{5}$ Midgut volvulus involves the entire bowel from the ligament of Treitz to the mid-transverse colon and the prognosis is very poor.

Sonographic findings of intestinal volvulus were first reported in $1983 .{ }^{6}$ Prenatal diagnosis and sonographic findings suggestive of in utero volvulus are important for multidisciplinary planning at the time of delivery. In addition to dilated loops of bowel, the "whirlpool sign" (bowel loops with accompanying mesentery and vessels wrapping around the main SMA) and the "coffee bean" or kinked loop sign (which is a distention of a very short segment of bowel, which resembles a coffee bean) are signs of pathognomonic for intestinal volvulus (-Fig. 3). ${ }^{7}$ Polyhydramnios has been a sign consistent with complete obstruction from intestinal volvulus. ${ }^{1}$ One case report recommended the use of Doppler velocimetry to assess for fetal anemia when dilated loops of bowel are noted in association with ascites on prenatal ultrasound and fetal intestinal volvulus is suspected. ${ }^{5}$ Fetal magnetic resonance imaging may be helpful in establishing the exact site of obstruction prenatally; however, its cost and sparse availability of required expertise make this imaging modality impractical for most pregnancies-especially as the diagnosis is

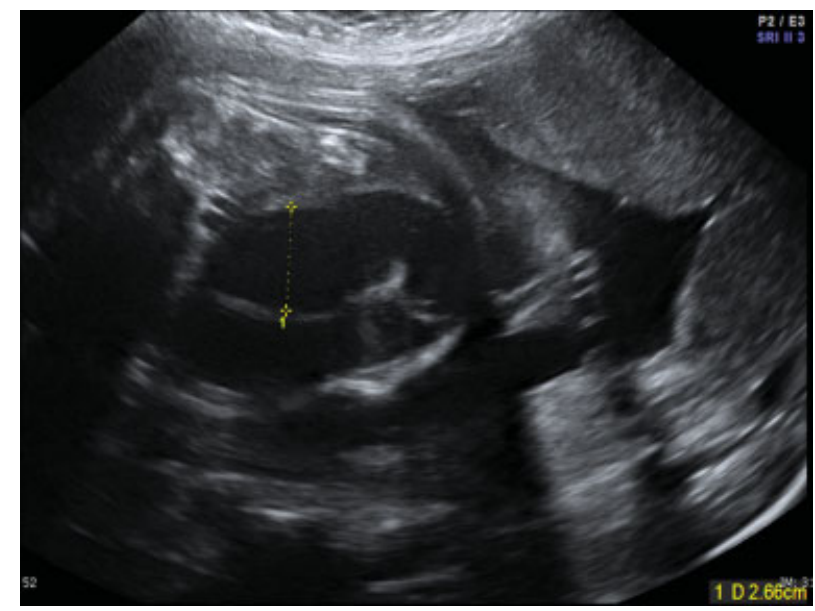

Fig. 3 "Coffee bean" sign at 36 weeks of gestation sonogram.

typically acutely suspected close to delivery. Postnatally, plain radiography is sufficient, and upper barium studies are rarely needed. ${ }^{8}$

The sonographic findings of fetal intestinal intussusception at term may include a "target-like" lesion; Shimotake reported that this is a round hyperechoic area of fetal bowel mucosa surrounded by relatively hypoechoic ring of bowel wall. ${ }^{9}$ Intussusception can lead to vascular impairment and bowel necrosis resulting in intestinal atresia; similarly, fetal intestinal volvulus may be complicated by ischemia and can lead to intestinal atresia. ${ }^{10}$ Nijagal et al propose sonographic examination of the umbilical cord in fetuses with intestinal atresia to identify those at risk of hemorrhage. ${ }^{11}$ Although our case did not reveal target-like lesion, umbilical cord ulceration, or fetal ascites on prenatal sonography, striking tubular dilatation of the digestive tract in third trimester suggested the development of fetal intestinal pathology (-Fig. 4).

Clinically, sluggish fetal movements or the observation of a nonreactive tracing on cardiotocography helps in reaching the diagnosis of volvulus. ${ }^{8}$ Fetal distress such as decreased fetal movement and minimal/absent short-term heart rate variability can be presenting signs, as in our

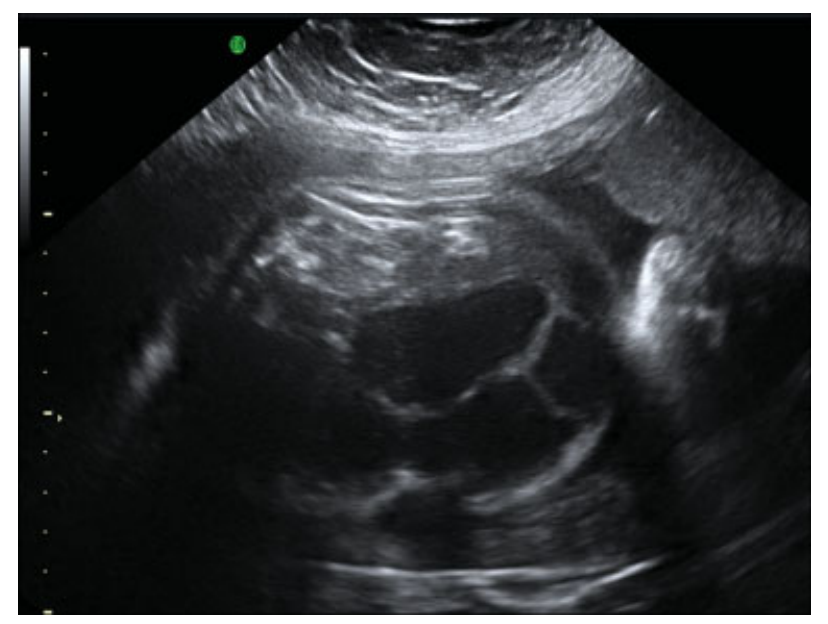

Fig. 4 Volvulus at 36 weeks of gestation showing striking tubular dilatation, suggested the development of fetal intestinal pathology. 


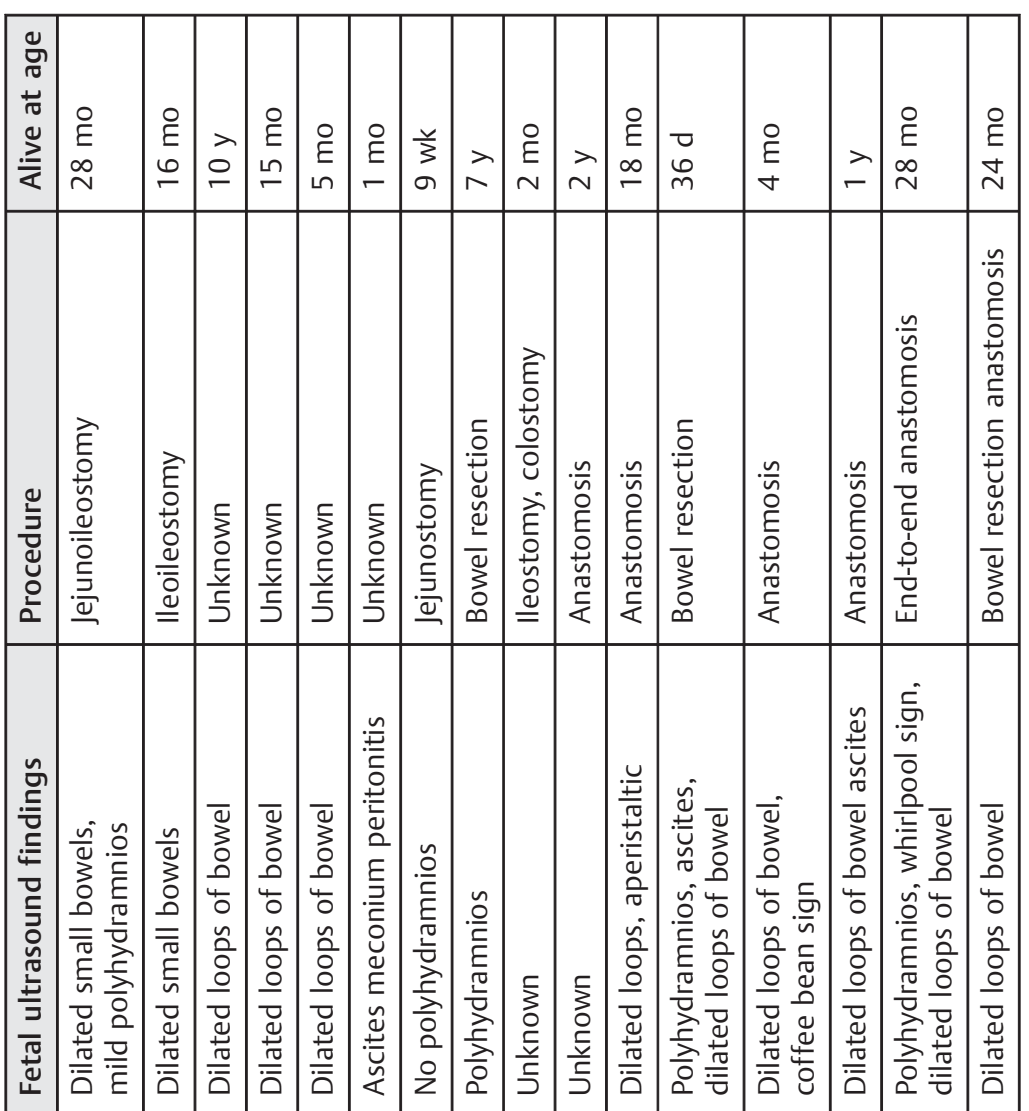

ত్ర

\begin{tabular}{|c|c|c|c|c|c|c|c|c|c|c|c|c|c|c|c|c|}
\hline 䓌 & $\frac{\stackrel{0}{n}}{\text { i }}$ & 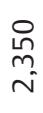 & $\begin{array}{l}\infty \\
\infty \\
i\end{array}$ & $\begin{array}{l}0 \\
\stackrel{0}{0} \\
i\end{array}$ & $\begin{array}{l}\stackrel{8}{\sigma} \\
\text { i }\end{array}$ & $\begin{array}{l}8 \\
\text { m }\end{array}$ & $\frac{s}{z}$ & 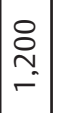 & $\frac{s}{z}$ & $\begin{array}{l}\stackrel{8}{ } \\
\infty \\
\sim\end{array}$ & \begin{tabular}{l|l}
2 & \\
$\vdots$ & \\
$i$ &
\end{tabular} & $\begin{array}{l}8 \\
\vdots \\
i\end{array}$ & $\begin{array}{l}\text { প } \\
\text { i } \\
i\end{array}$ & $\mid \begin{array}{l}0 \\
\infty \\
0 \\
--\end{array}$ & $\begin{array}{l}\stackrel{8}{\circ} \\
\text { i }\end{array}$ & $\stackrel{\substack{\stackrel{m}{m} \\
m}}{m}$ \\
\hline$\stackrel{\rtimes}{\sim}$ & L & $\Sigma$ & L & L & L & ப & $\supset$ & L & $\supset$ & $\Sigma$ & $\Sigma$ & $\Sigma$ & $\Sigma$ & $\Sigma$ & L & $\Sigma$ \\
\hline 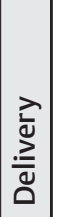 & 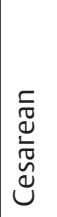 & 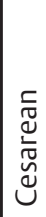 & 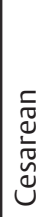 & 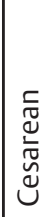 & 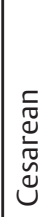 & 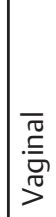 & 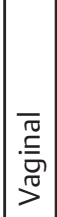 & 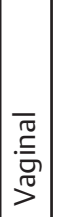 & 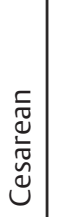 & 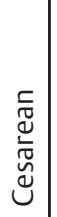 & 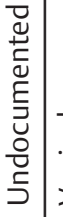 & 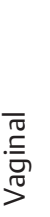 & 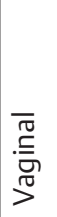 & 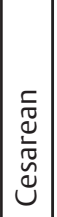 & 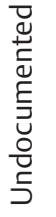 & 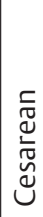 \\
\hline $\begin{array}{l}\frac{5}{3} \\
\text { gu } \\
\text { ğ }\end{array}$ & $\tilde{m}$ & $\ddot{m}$ & $\stackrel{m}{m}$ & m & m & m & $\hat{m}$ & $\begin{array}{l}0 \\
\hat{1} \\
\grave{N}\end{array}$ & $\vec{m}$ & $\stackrel{m}{m}$ & $\dot{m}$ & m & $\stackrel{m}{m}$ & $\bar{m}$ & $\hat{m}$ & $\hat{m}$ \\
\hline $\begin{array}{l}\text { ते } \\
\text { जे }\end{array}$ & 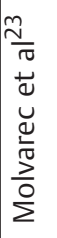 & 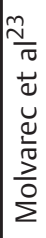 & 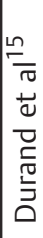 & 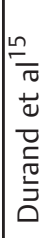 & 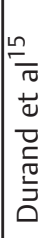 & 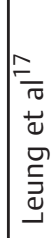 & 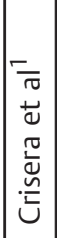 & 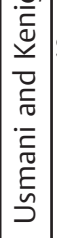 & 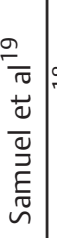 & 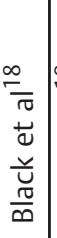 & $\begin{array}{l}\infty \\
\frac{\infty}{\pi} \\
\frac{\tilde{U}}{\tilde{U}} \\
\underline{\underline{u}} \\
\frac{\pi}{\infty}\end{array}$ & 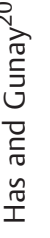 & 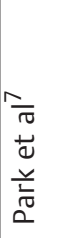 & 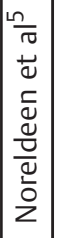 & $\begin{array}{l}\bar{v} \\
\frac{\pi}{v} \\
\stackrel{0}{\nu} \\
\nu\end{array}$ & 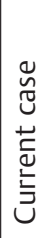 \\
\hline 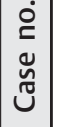 & - & $\sim$ & $m$ & $\sigma$ & เn & 6 & $n$ & $\infty$ & $\sigma$ & 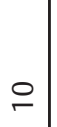 & $=?$ & $\simeq$ & $\stackrel{m}{n}$ & $\nabla$ & $\stackrel{n}{\stackrel{n}{2}}$ & $\stackrel{0}{\circ}$ \\
\hline
\end{tabular}


case. ${ }^{5}$ Witter and Molteni discuss a case of fetal small bowel volvulus that leads to massive hemoperitoneum presenting with decreased fetal movement and a positive oxytocin challenge test. ${ }^{1,12}$

Reported cases of fetal intestinal volvulus have described infants delivered abdominally or vaginally. Interestingly, there has been a postulated relationship between volvulus and preterm delivery, suggesting that acute fetal distress may activate the adrenal and hypothalamic hormones and induce premature uterine activity and birth. ${ }^{1}$ This is consistent with the reported cases that were prematurely delivered in their great majority.

Survival of these infants is influenced by the gestational age at birth, size of compromised bowel, and the ability to tolerate neonatal surgery. Although not the most common outcome, stillbirth has been reported in the literature in cases of fetal volvulus. To our knowledge, there have been three reported cases of term fetal demise. In 2004, Allahdin and Kay report a case of fetal demise at 39 weeks of gestation. Autopsy revealed ischemic hemorrhagic necrosis secondary to intestinal volvulus that was not suspected prenatally. ${ }^{13}$ Subsequently in 2007, Trachsel et al reported a case of fetal demise at 38 weeks of gestation; autopsy in that case revealed intestinal volvulus with extended hemorrhagic infarction of the small bowel but no perforation. ${ }^{14}$ The fetus was notably affected by cystic fibrosis-characterized by viscous mucous secretions that can obstruct the bowel lumen, thus predisposing to volvulus. In contrast, Durand et al reported a case of perinatal survival at 39 weeks of a fetus with compound heterozygosity for mutations at $\Delta \mathrm{F} 508$ and Y1092X. ${ }^{15}$ Steffensen et al reported a case of fetal demise at 38 weeks of gestation, due to cardiovascular failure and shock from midgut volvulus; the fetus also had intestinal atresia and arthrogryposis. ${ }^{6}$ Lee et al reported a case at 38 weeks of fetal ileal atresia and volvulus; however, it is not clear if that infant survived the end-ileostomy repair. ${ }^{16}$ Various etiologies for intestinal volvulus have been described in infants alive in the neonatal period, including meconium peritonitis and intestinal mesentery defects. ${ }^{17,18}$ Pathognomonic sonographic findings in the third trimester ultrasound examination, such as the whirlpool sign, aids in rapid antenatal diagnosis of intestinal volvulus. ${ }^{19-21}$ Characteristics of these cases are summarized in - Table $\mathbf{1}$.

Currently, the American College of Obstetricians and Gynecologists and the American College of Radiology recommend routine sonograms in the first and/or second trimester, but state that no evidence exists to recommend routine sonograms in the third trimester. ${ }^{22}$ Our case involves fetal volvulus, intussusception, and intestinal atresia with perinatal and long-term survival of a term infant. Cases reviewed of survival of fetal intestinal volvulus demonstrate that prenatal diagnosis and neonatal anticipation affect outcomes decisively. Molvarec et al reported two cases of fetal intestinal volvulus, which were delivered promptly on the basis of ultrasound findings and biophysical monitoring of the fetus. ${ }^{23}$ Bawa and Kannan demonstrate different outcomes of fetal intestinal volvulus based on the performance of prenatal scanning; one fetus identified with dilated bowel underwent prompt surgical intervention and survived, whereas another succumbed with the postnatal diagnosis of volvulus when no prenatal sonogram was performed. ${ }^{24}$ They suggested that a single third trimester scan for fetal anomalies may be an effective strategy to reduce perinatal mortality. ${ }^{24}$

Sonographic detection of dilated fetal bowel usually represents a normal variant without clinical significance. Cystic fibrosis (meconium ileus), intestinal obstruction/atresia, and cloacal malformations make up the majority of pathological conditions associated with dilated fetal bowel; these conditions should also prompt prenatal pediatric surgery and genetics consultations and management in a tertiary care center. For the general obstetrician gynecologist, it is important to note that severe and abrupt fetal bowel dilatation in a previously normal fetus with hypoperistalsis and poor fetal activity are strongly associated with fetal volvulus. This can result in a poor prognosis for perinatal outcome without prompt and appropriate intervention.

The main barrier to routine third trimester sonography is cost-effectiveness-there is no doubt that some urological and intestinal anomalies would be identified with likely improvement of perinatal outcomes, but the cost of screening the entire population in the third trimester renders this approach unattractive from a public health perspective. Our case and the related literature suggest that particular attention should be paid to the fetal digestive tract at the time of fetal growth sonograms after 32 weeks. In a society of delayed childbearing, evolving obesity epidemic and associated comorbidities (chronic hypertension, diabetes mellitus), as many as two-thirds of all pregnant women will have an indication for a third trimester scan-we suggest that this is an opportunity to screen for fetal intestinal anomalies that could substantially affect perinatal care and avert late fetal loss or serious neonatal morbidity. The new onset of striking tubular intestinal dilatation in the third trimester in combination with clinical suspicion for fetal intestinal volvulus should prompt multidisciplinary consultation and planning with neonatology and pediatric surgery to help optimize perinatal outcomes in a tertiary care hospital.

\section{References}

1 Crisera CA, Ginsburg HB, Gittes GK. Fetal midgut volvulus presenting at term. J Pediatr Surg 1999;34(8):1280-1281

2 Hsu CY, Chiba Y, Fukui O, Sasaki Y, Miyashita S. Counterclockwise barber-pole sign on prenatal three-dimensional power Doppler sonography in a case of duodenal obstruction without intestinal malrotation. J Clin Ultrasound 2004;32(2):86-90

3 Gaikwad A, Ghongade D, Kittad P. Fatal midgut volvulus: a rare cause of gestational intestinal obstruction. Abdom Imaging 2010;35(3):288-290

4 Usmani SS, Kenigsberg K. Intrauterine volvulus without malrotation. J Pediatr Surg 1991;26(12):1409-1410

5 Noreldeen SA, Hodgett SG, Venkat-Raman N. Midgut volvulus with hemorrhagic ascites: a rare cause of fetal anemia. Ultrasound Obstet Gynecol 2008;31(3):352-354

6 Steffensen TS, Gilbert-Barness E, DeStefano KA, Kontopoulos EV. Midgut volvulus causing fetal demise in utero. Fetal Pediatr Pathol 2008;27(4-5):223-231 
7 Park JS, Cha SJ, Kim BG, et al. Intrauterine midgut volvulus without malrotation: diagnosis from the 'coffee bean sign'. World J Gastroenterol 2008;14(9):1456-1458

8 Rajab KE, Al Juffairi Z, Issa AA. Antenatal diagnosis and management of fetal mid-gut volvulus. Bahrain Med Bull 2007;29: 106-108

9 Yang JI, Kim HS, Chang KH, Hong J, Joo HJ, Ryu HS. Intrauterine intussusception presenting as fetal ascites at prenatal ultrasonography. Am J Perinatol 2004;21(4):241-246

10 Yoo SJ, Park KW, Cho SY, Sim JS, Hhan KS. Definitive diagnosis of intestinal volvulus in utero. Ultrasound Obstet Gynecol 1999; 13(3):200-203

11 Nijagal A, Rand L, Goldstein R, Poder L, Miniati D. Intrauterine umbilical cord hemorrhage with associated jejunal atresia captured by real-time ultrasound. Am J Obstet Gynecol 2009;200(3): e5-e6

12 Witter FR, Molteni RA. Intrauterine intestinal volvulus with hemoperitoneum presenting as fetal distress at 34 weeks' gestation. Am J Obstet Gynecol 1986;155(5):1080-1081

13 Allahdin S, Kay V. Ischaemic haemorrhagic necrosis of the intestine secondary to volvulus of the midgut: a silent cause of intrauterine death. J Obstet Gynaecol 2004;24(3):310

14 Trachsel D, Heinimann K, Bösch N, Hammer J. Cystic fibrosis and intrauterine death. J Perinatol 2007;27(3):181-182

15 Durand M, Coste K, Martin A, et al. Fetal midgut volvulus as a sign for cystic fibrosis. Prenat Diagn 2008;28(10):973-974
16 Lee JH, Im SA, Lee G. Evolution of sonographic findings in a fetus with ileal atresia. J Clin Ultrasound 2011;39(6):359-362

17 Leung WC, Pandya P, Seaward G, Windrim R, Ryan G. Changes in fetal heart rate pattern after intrauterine paracentesis in one twin with meconium peritonitis. Prenat Diagn 2001;21(8):693-696

18 Black PR, Mueller D, Crow J, Morris RC, Husain AN. Mesenteric defects as a cause of intestinal volvulus without malrotation and as the possible primary etiology of intestinal atresia. J Pediatr Surg 1994;29(10):1339-1343

19 Samuel N, Dicker D, Feldberg D, Goldman JA. Ultrasound diagnosis and management of fetal intestinal obstruction and volvulus in utero. J Perinat Med 1984;12(6):333-337

20 Has R, Gunay S. 'Whirlpool' sign in the prenatal diagnosis of intestinal volvulus. Ultrasound Obstet Gynecol 2002;20(3):307-308

21 Yu W, Ailu C, Bing W. Sonographic diagnosis of fetal intestinal volvulus with ileal atresia: A case report. J Clin Ultrasound 2013; 41(4):255-257

22 American College of Obstetricians and Gynecologists. ACOG Practice Bulletin No. 101: Ultrasonography in pregnancy. Obstet Gynecol 2009;113(2, Pt 1):451-461

23 Molvarec A, Bábinszki A, Kovács K, Tóth F, Szalay J. Intrauterine intestinal obstruction due to fetal midgut volvulus: a report of two cases. Fetal Diagn Ther 2007;22(1):38-40

24 Bawa M, Kannan NL. Even a single third trimester antenatal fetal screening for congenital anomalies can be life saving. Indian J Pediatr 2010;77(1):103-104 